\section{Fazialisparese \\ Was Sie bei Gesichtslähmung zuerst veranlassen sollten}

Von F. X. Glocker

Eine 70-jährige Patientin bemerkt morgens nach dem Aufstehen ein Brennen im linken Auge. Beim Frühstück läuft ihr der Kaffee den linken Mundwinkel herunter und die linke Gesichtshälfte fühlt sich „taub“ an. Im Bereich des linken Ohres spürt sie ein leichtes Druckgefühl. Bisher ist die Patientin immer gesund gewesen und es bestehen bei ihr keine Gefäßrisikofaktoren. Ein Schlaganfall lässt sich in der Anamnese nicht eruieren.

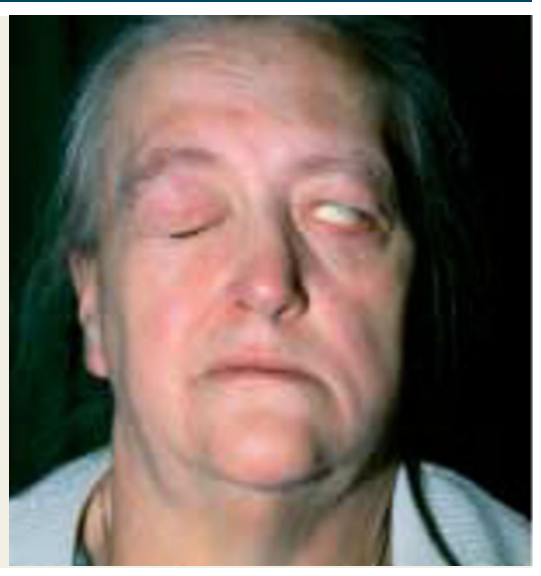

— Zuerst müssen Sie klären, ob es sich um eine periphere oder zentrale Lähmung handelt.

- Periphere Gesichtslähmung (durch Schädigung im Kerngebiet oder peripheren Nervenverlauf): Hier erscheint die Stirnpartie faltenarm bis faltenlos, der Lidschluss ist defizient, Mundspitzen und Mundwinkelhebung sind eingeschränkt und die Nasolabialfalte erscheint verstrichen.

- Zentraler Lähmungstyp: Hier ist überwiegend die Mund- und Wangenmuskulatur betroffen, die Stirnmuskeln bleiben aufgrund der bilateralen supranukleären Versorgung intakt, der Lidschluss ist bei Prüfung komplett. Zentrale Fazialisparesen sind immer verdächtig auf einen Schlaganfall und als medizinischer Notfall zu behandeln.

\section{Wie gehen Sie weiter vor?}

- Mit ca. 80\% häufigste Ursache der peripheren Gesichtslähmung ist die idiopathische Fazialisparese. Es werden regelmäßig Schmerzen im Bereich des Ohres angegeben. Eine sehr ausgeprägte Schmerzsymptomatik sollte jedoch immer an eine Zosterinfektion denken lassen. Gelegentlich sind die Herpesbläschen ausschließlich im Gehörgang nachweisbar. Deshalb muss jeder Patient otoskopiert werden.

- Eine Geschmacksstörung ist bei ca. der Hälfte der Fälle nachweisbar, eine Hörstörung im Sinne einer Hyperakusis ist wesentlich seltener. Zwischen einem Drittel und der Hälfte der Patienten entwickeln eine vollständige Gesichtslähmung. Die gelegentliche Angabe von „Sensibilitätsstörungen“ im Gesichtsbereich kann Folge der ver- änderten Muskelinnervation sein. Eine zeitnahe Vorstellung des Patienten bei einem neurophysiologisch tätigen Neurologen ist anzustreben, da in den ersten 72 Stunden nach Symptombeginn mittels elektrophysiologischer Technik (Magnetstimulation) relativ sicher zwischen der harmlosen idiopathischen Genese und anderen Ursachen differenziert werden kann.

- Ist diese nicht möglich oder verfügbar, so ist eine serologische Diagnostik auf Herpes simplex Typ I, Varizellen und Borrelien sowie eine Liquoruntersuchung indiziert. Anhaltspunkte, die an einer idiopathischen Genese zweifeln lassen, sind in Tabelle 1 zusammengefasst.

\section{Gesichtslähmung bei Kindern}

Bei Kindern ist eine routinemäßige Borrelienserologie und Lumbalpunktion zu empfehlen, da hier bei ca. 40\% aller Fälle eine monosymptomatische Borrelieninfektion als Ursache der Gesichtslähmung gefunden wird.

\section{Tabelle 1}

Untypische Symptome/ Befunde für eine idiopathische Fazialisparese

- Anhaltende Schmerzen (retro-)aurikulär, Tragusschmerz

- Bläschen (evtl. nur im Gehörgang)

- Zervikalgien

- Sensibles Defizit

- Schwindel

_ Einseitige Hörstörung/Tinnitus

- Parotisschwellung/-resistenz

\section{Therapie}

- In der Annahme eines Pathomechanismus mit ödematöser Schwellung des Nerven und hieraus resultierender Druckschädigung ist bei der idiopathischen Fazialisparese eine frühestmögliche Therapie mit Steroiden entscheidend. Es können 2 x 25 mg Prednison/d oder alternativ einmal täglich 1-1,5 mg/kg KG gegeben werden.

- Nach derzeitigem Erkenntnisstand ist eine Kombination der Steroidtherapie mit einem Virustatikum bei der idiopathischen Fazialisparese aus medizinischer und ökonomischer Sicht nicht gerechtfertigt.

- In der Akutphase kommt der Vermeidung von Hornhautulzerationen mittels Augensalbe und bei inkomplettem Augenschluss das Tragen eines Uhrglasverbandes oder einer Silikonschale eine wesentliche Bedeutung zu.

\section{Verlauf und Prognose}

Eine gute Rückbildung der Parese, beginnend innerhalb von vier bis sechs Wochen nach Erkrankungsbeginn, erlangen 80-90\% der Patienten. Bei den verbleibenden Patienten kann bis zum Ablauf von einem Jahr noch eine Besserung eintreten. Diese führt dann meist zu einer subjektiv beeinträchtigenden Defektheilung. Rezidive treten bei 5-10\% der Fälle auf.

\section{Anschrift des Verfassers:}

Prof. Dr. med. Franz X. Glocker, MediClin Seidel-Klinik Bad Bellingen, Zentrum für Rheumatologie, Wirbelsäulenleiden und Neuromuskuläre Erkrankungen,

Hebelweg 4, D-79415 Bad Bellingen, E-Mail: franz.glocker@uniklinik-freiburg.de 